\title{
Actinomycosis of the urinary bladder
}

\author{
Chun Huang; ${ }^{*}$ Turki Al-Essawi, MD, FRCSC ${ }^{\dagger}$ \\ *College of Medicine, University of Saskatchewan, Saskatoon, SK; 'Faculty of Medicine, Division of Urology, University of Manitoba, Winnipeg, MB
}

Cite as: Can Urol Assoc J 2013;7(7-8):e502-4. http://dx.doi.org/10.5489/cuaj.405

Published online on July 2, 2013.

\section{Abstract}

Actinomycosis of the urinary bladder is a rare anaerobic bacterial infection caused by Actinomyces isrealii. Initial diagnosis is often difficult and this disease is easily misdiagnosed as a urothelial or urachal tumour. The definitive diagnosis is usually made postoperatively via tissue pathology. We discuss a case of a 54-year-old male with a smoking history and a 2.5-week history of gross hematuria. Ultrasound, computed tomography and cystoscopy revealed a large inflammatory mass adherent to the right, anterior bladder wall, suggesting malignancy. Transurethral resection and histological pathology subsequently confirmed inflammatory urothelium and gram-positive bacteria consistent with actinomyces species.

\section{Introduction}

Actinomycosis of the urinary bladder is a rare infection caused by the anaerobic bacteria Actinomyces isrealii. Initial diagnosis is often difficult, as it mimics urothelial or urachal tumours. ${ }^{1,2}$ The definitive diagnosis is usually made postoperatively via tissue pathology. We report a case of primary bladder actinomycosis initially diagnosed as a urothelial carcinoma that subsequently underwent a transurethral resection of a bladder tumour (TURBT). Pathologic examination subsequently confirmed actinomycosis.

\section{Case report}

A 54-year-old male presented with symptoms of 2.5 weeks of painless, gross hematuria, frequency, nocturia and hesitancy. He denied any history of irritative voiding symptoms. He has a medical history of diabetes, dyslipidemia, erectile dysfunction and a 10-pack/year smoking history. On physical exam, he had a benign abdominal and rectal exam.
Follow-up ultrasound by the family physician revealed a $7.7 \times 5.7 \times 7.3 \mathrm{~cm}$ mass extending from the right bladder wall with vascularity on colour Doppler and possible extension through the bladder wall (Fig. 1). Cystoscopy showed a large bladder tumour involving the posterior wall. Urothelial carcinoma and urachal tumours were suspected at this time. The urine cytology was negative for malignant cells. A transurethral resection of the mass showed a high-grade invasive lesion macroscopically, but tissue diagnosis returned inflamed urothelial mucosa with colonies of gram positive bacteria morphologically consistent with actinomyces species (Fig. 2).

Based on the pathology report, a computed tomography (CT) scan was ordered to assess for the extent of infection, inflammation and lymph node involvement. Imaging showed extensive inflammatory thickening of the bladder wall and broad-based mass on the right, anterior wall with perivesical fat involvement tracking in a cephalad direction (Fig. 3).

Treatment with high-dose amoxicillin was carried out for 3 months. Follow-up CT and cystoscopy post-treatment showed residual mild inflammation and edema on the right posterior bladder wall with vast improvement over previous investigations (Fig. 4). Another 3-month regimen of amoxicillin was prescribed with instructions for follow-up imaging.

\section{Discussion}

\section{Pathophysiology}

Actinomycosis is a chronic infectious disease with widespread anatomical distribution. The varied presentations of this disease are often concluded to be malignancy rather than infection, leading it to be considered the most misdiagnosed disease. The offending organism is A. israelii, a gram-positive, anaerobic bacilli, and is considered part of the normal flora of the gastrointestinal tract in humans. ${ }^{3,4}$ Overall, infection of the genitourinary system is rare, with only a few reported cases. ${ }^{2,5,6}$ 


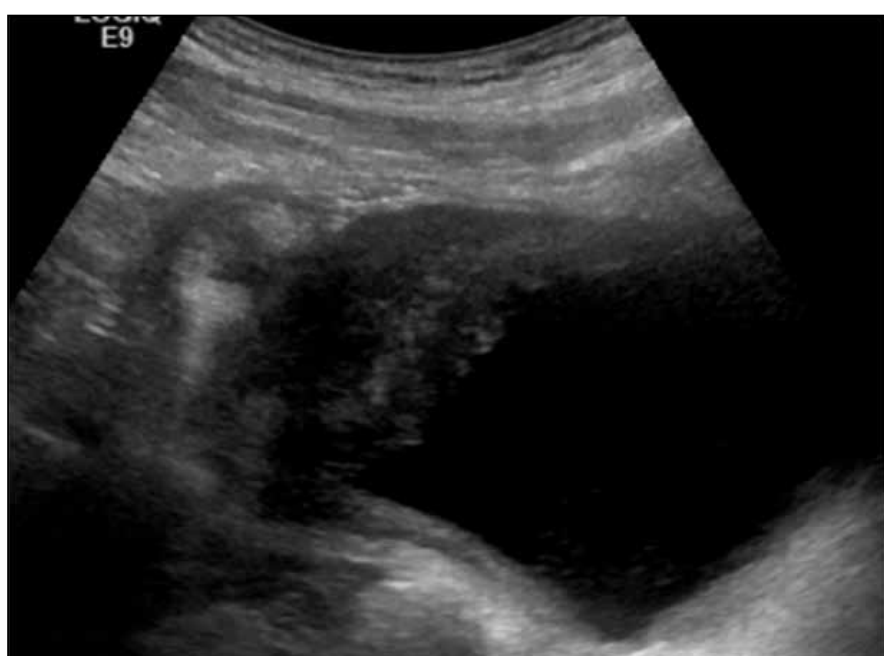

Fig. 1. Ultrasound image of the $7.7 \times 5.7 \times 7.3 \mathrm{~cm}$ mass extending from the right bladder wall. There appears to be perivesicular involvement which is highly suspicious for a high-grade tumour, such as a urothelial or urachal neoplasm.

The bacterium itself is not considered pathogenic in nonimmunocompromised individuals, but circumstantial evidence suggests that mucosal trauma and infection by other organisms permits opportunistic invasion. ${ }^{3}$ The progression of infection is slow. Common symptoms of bladder actinomycosis include hematuria, irritative voiding symptoms and lower abdominal pain. These can be accompanied by constitutional symptoms and mass-effect, such as obstruction or visceral pressure sensation. ${ }^{7,8}$ Due to non-specific symptom profiles, clinical diagnosis of actinomycosis is made with difficulty.

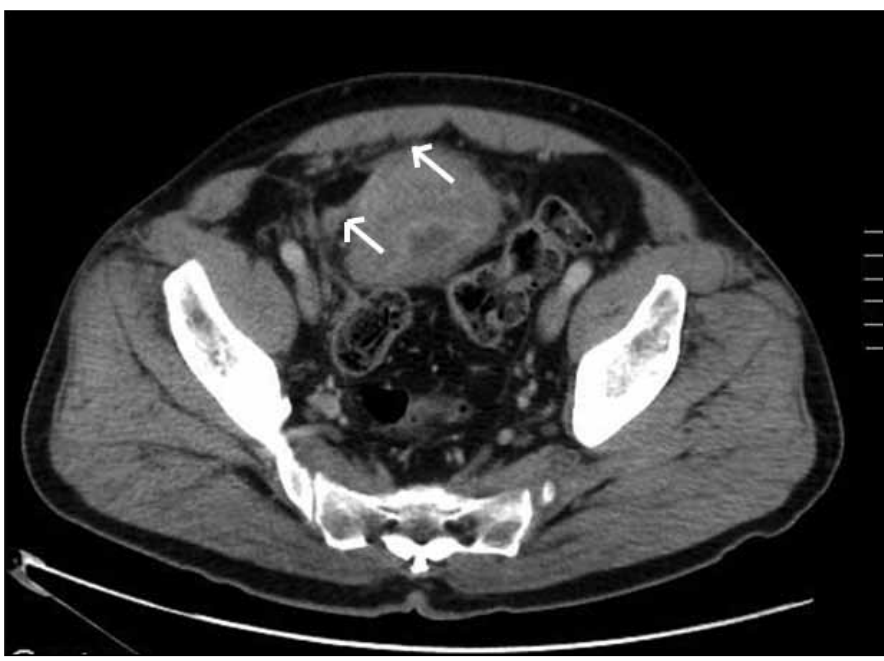

Fig. 3. A computed tomography slice prior to transurethral resection showing thickened bladder wall as well as the broad base of the original mass. There is also possible perivesicle involvement as marked by the white arrows.

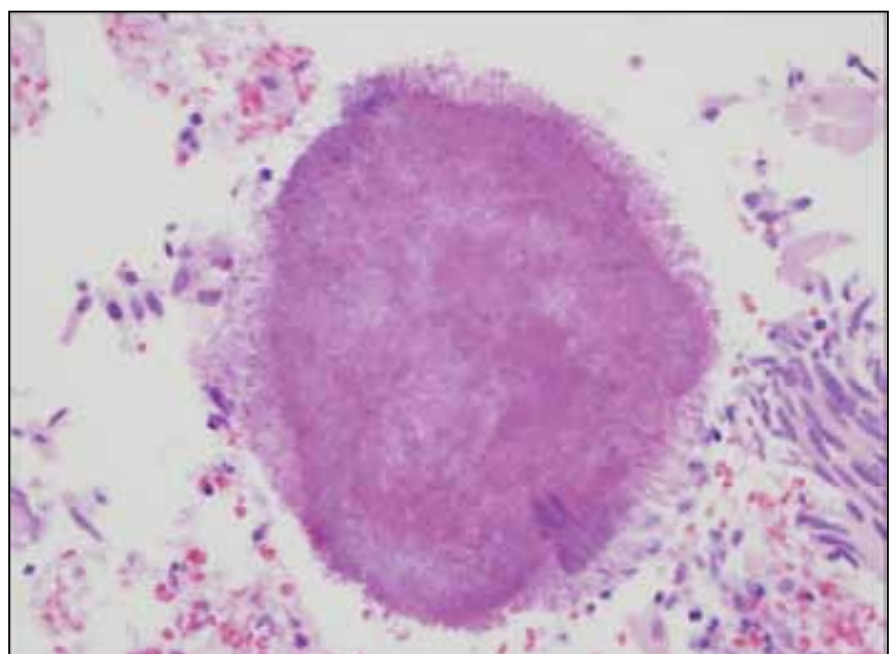

Fig. 2. The gram stain from our case showing a "sulphur granule" that is typically associated with actinomycosis infections. They are not actually sulphur containing but appear similar.

\section{Diagnosis}

Diagnosis of vesicular actinomycosis is often delayed due to the process of investigating the possibility of a urothelial malignancy, with vesicular and urachal neoplasms carrying greater indices of suspicion. Sulfur granules are often seen in biopsy specimens, but may also be detected in urine cytology as an initial clue to the etiology. ${ }^{7,9}$ Microscopic analysis of tissue should demonstrate Gram positive bacilli with the appearance of filaments and hypea. Inflammatory tissue change should also be present. ${ }^{9,10}$ Definitive diagnosis requires bacterial cultures or histological identification of the microbiota in question.

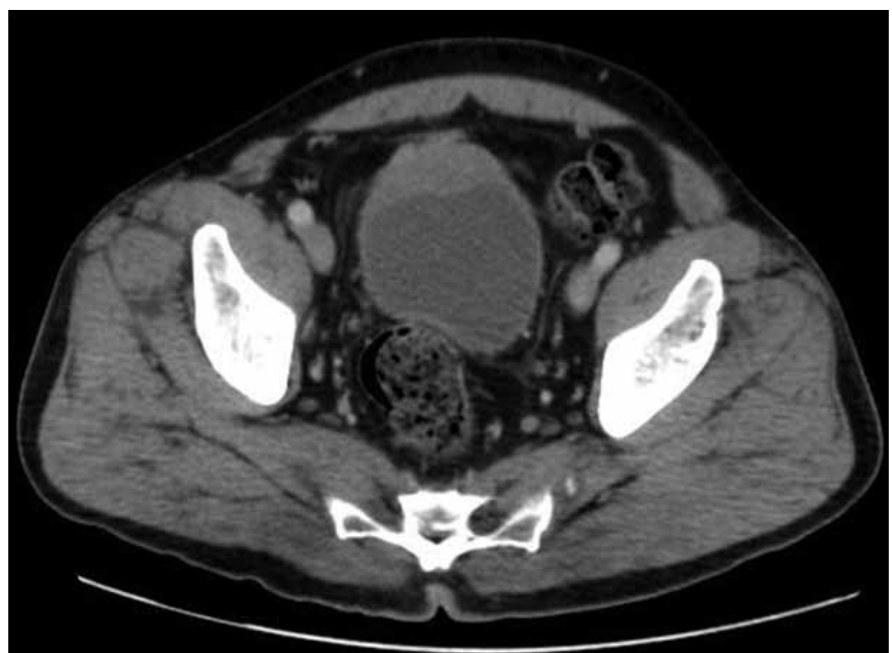

Fig. 4. The 3-month follow-up computed tomography post-transurethral resection of the bladder tumour and antibiotic treatment. There is improvement of the bladder wall as it is no longer globally thickened. As well, the mass is reduced in size and the perivesicle tissue change has also resolved. 


\section{Treatment}

As its symptom profile is similar to malignancy, pelvic actinomycosis is often initially treated surgically or biopsied based on preoperative imaging results. Some authors suggest that definitive treatment of urinary bladder actinomycosis involves partial or total cystectomy. ${ }^{10}$ These treatments tend to be carried out in patients where transurethral resection was not attempted based on the cystoscopic and radiologic morphology. 3,7

Chronic antibiotic treatment of actinomycosis with penicillin as the drug of choice is the prevailing recommendation, ${ }^{1,6}$ especially when diagnosis is made preoperatively from TURBT pathology reports. Starting doses should be between 10 and 20 million units intravenously for 4 to 6 weeks. When combined with surgical excision or debridement, 1 to 2 million units, orally, over 12 to 18 months is indicated. Patients with penicillin allergies may be alternatively treated with doxycycline or erythromycin. ${ }^{7,9,10}$

\section{Conclusion}

Actinomycosis is a difficult infection to recognize and requires long-term management with antibiotics. Based on our experience, urothelial actinomycosis can be adequately followed up by repeat imaging or cystoscopy for efficacy of treatment.
Competing interests: None declared.

This paper has been peer-reviewed.

\section{References}

1. Dhamborvorn T, Tritipsatit S, Meemongkoldilok S. Actinomycosis of the urinary bladder. J Med Assoc Thai 2001;84:109-12.

2. Aguirrebengoa K, Arruza A, Bereciartua E, et al. Primary actinomycosis of the urinary bladder. Scand J Infect Dis 2000;32:330-1. http://dx.doi.org/10.1080/00365540050166054

3. Garner JP, Macdonald M, Kumar PK. Abdominal actinomycosis. Int I Surg 2007;5:441-8.

4. Margolin HN. Fungal Infections of the Urinary Tract. Semin Roentgenol 1971;6:323-30. http://dx.doi.org/10.1016/0037-198X(71)90042-3

5. Bottai A, Li Marzi V, Alessandrini $M$, et al. Intrauterine device-associated actinomycosis of the ovary and urinary bladder: a case report. Urol Int 2010;85:242-4. http://dx.doi.org/10.1159/000316069

6. Kirova $Y M$, Feuilhade $F$, Belda-Lefrère $M A$, et al. Intrauterine device-associated pelvic actinomycosis: a rare disease mimicking advanced ovarian cancer: a case report. Eur J Gynaecol Oncol 1997;18:502-3.

7. Marella VK, Hakimian 0, Wise GJ, et al. Pelvic Actinomycosis. Urologic Perspective. Int Braz I Urol 2004:30:367-76.

8. Talwar, OP, Ghosh A, Pradhan $S$, et al. A prospective and retrospective study of actinomycosis in last five years at Manipal Teaching Hospital, Pokhara, Nepal. Kathmandu Univ Med J (KUMJ) 2007:5:488-91.

9. Lee, K.R., Y.S. Ko, YU JW, et al. Primary Vesical Actinomycosis: A Case Diagnosed by Multiple Transabdominal Needle Biopsies. J Korean Med Sci 2002;17:121-4.

10. Wagenlehner FME, Mohren B, Naber KG, et al. Abdominal actinomycosis: Concise communications. Clinical Microbiology \& Infection 2003;9:881-5.

Correspondence: Dr. Turki Al-Essawi, AD203-820 Sherbrook St, Health Sciences Centre, Winnipeg, MB R3A 1R9; fax: 204-787-3040; talessawi@hsc.mb.ca 